\title{
Statistical description of thermoreversible chemical bonding in metallic alloys
}

\author{
R.E. Ryltsev ${ }^{1, a}$, L.D. Son ${ }^{2}$, and V.E. Sidorov ${ }^{2}$ \\ 1 Institute of Metallurgy of Ural Division of Russian Academy of Sciences, Amudsena str. 101, 620016 Russia \\ 2 Ural State Pedagogical University, pr. Kosmonavtov 26, Yekaterinburg, 620017 Russia
}

\begin{abstract}
A general statistical model, developed in our previous works for describing network-forming systems, have applied to study of metallic alloys with chemical bonding. The model is based on the representation of the partition function for all possible configurations of a thermoreversible network in the form of a functional integral over a scalar field. The main object of our investigation was the solution of free atoms $A, B$ and the elements of chemical short-range order with the stoichiometry $A_{2} B$ that could form thermoreversible chemical bonds. The thermodynamic and structural properties of this system is analyzed. It is revealed that at some temperatures and concentrations, geometry percolation of the network of chemical bonds and thus a sol-gel transition may take place. The critical percolation line is plotted in common with phase diagram. Three different scenarios of sol-gel transition is considered in light of competition between gelation and phase separation phenomena.
\end{abstract}

\section{Introduction}

Network-forming systems are among the most popular objects in the physics of condensed matter. On the one hand, they are widespread in nature and have been extensively used in technology, and the other hand, they exhibit a nontrivial complex behavior, which is of general scientific interest. One of the most universal and interesting phenomena characteristic network-forming systems is the sol-gel transition. This transition is considered to mean the formation of an infinite percolation cluster that consists of some structural units of the material. If the bonds between these structural units are thermoreversible (i.e. they have an energy $U \sim k T$ and so a finite lifetime), the case in point is a physical gel. Among physical gels, there are a large number of systems of different nature: tetrahedral liquids $\left(\mathrm{H}_{2} \mathrm{O}, \mathrm{SiO}_{2}, \mathrm{GeO}_{2}\right)$ and they solutions [1], [2], organic liquids [3], associating [4] an supramolecular [5], [6] polymers and many other systems [7]. The systems mentioned are very popular objects of research and they have been intensively studied by mane authors for several decades.

But there is a wide class of systems whose gel-forming properties are investigated relatively poorly. These are alloys with pronounced chemical short-range order (CSRO) expressed by well-defined stable clusters caused by strong chemical bonding between atoms. The elements of such CSRO are able to couple by directed bonding, so strong tendency to percolation network formation takes place [1]. Note, that such percolation cluster has finite lifetime and exists under certain conditions. That is similar to formation of thermoreversible gels in the aforementioned sys-

\footnotetext{
a e-mail: rrylcev@mail.ru
}

tems. So one can use the term "thermoreversible gelation" in this case also.

The aim of this work is to investigate the alloys with CSRO in light of gelation phenomenon. As supposed in [16], [17], metal-metalloid and Al-REM are such alloys, so these cases are concerned below. To describe such systems, we make the following model assumptions (see [16], [17] for physical background):

- there are elements of CSRO (hereafter referred to as associates) with stoichiometry $A_{2} B$ in the alloy, where $A$ is either a transition metal or aluminium, and $B$ is either a metalloid or a rare earth respectively;

- the lifetime of the associates $\tau_{\text {chem }}$ is significantly greater than the period of thermal fluctuations, thus one treats them as relatively rigid units on the time scale $t \ll$ $\tau_{\text {chem }}$;

- these associates are able to couple by directed and saturated bonding, and the maximum number of bonds per an associate is equal to 4 . Thus, they serve as structural "bricks" for network building just as for molecular liquids;

- the coupled associates forms a percolation cluster under certain conditions.

In order to realize these assumptions, one needs the statistical model of a gel-forming systems with directed and saturated bonds between structural units. A great number of models based on different approaches have been proposed for this aim [8], [9], [10], [11], [12], [13], [14]. The majority of these models include various phenomenological approximations regarding the structure of clusters before and after the percolation transition. Because of different initial assumptions, the aforementioned models have

This is an Open Access article distributed under the terms of the Creative Commons Attribution-Noncommercial License 3.0, which permits unrestricted use, distribution, and reproduction in any noncommercial medium, provided the original work is properly cited. 
led to different results for thermodynamic and structural properties of gel-forming systems.

In this paper we propose a statistical model that does not include prior assumptions about the structure of clusters and can be treated as a universal tool for investigating network-forming systems. The model is based on the mathematically rigorous representation of the partition function for the system of thermoreversible chains with arbitrary nature and configuration in the form of a functional integral over a scalar field. This approach was proposed in our previous work [15] for molecular liquids. The subsequently this formalism has been applied to the description of the thermodynamic and structural properties of binary glass-forming alloys [16], [17]. In our recent paper [18] we have developed mathematical methods for describing sol-gel transition in the frames of this approach and applied them for investigation of polymer-like binary systems whose structural gel-forming units are stable molecules that have functional groups forming thermoreversible chemical bonds. This work is the next step on this way: we will apply developed methods to describe gel-forming systems whose structural units are the elements of CSRO with finite lifetime.

\section{Formulation of the model}

Our model can be formulated in the following way. Let us consider a binary alloy A-B which also contains the associates with the stoichiometry $A_{p} B_{q}(p, q=0,1,2, \ldots)$.

We suppose that the interaction energy between associates depends on their mutual orientation. Thus the pair potential of two associates $\alpha$ and $\beta$ located at the points $\mathbf{r}$ and $\mathbf{r}^{\prime}$ respectively can be written as

$$
V_{\alpha \beta}\left(\mathbf{r}, \mathbf{r}^{\prime}\right)=V_{\alpha \beta}^{(c e n t)}\left(\mathbf{r}-\mathbf{r}^{\prime}\right)+V_{\alpha \beta}^{(\text {orient })}\left(\Omega(\mathbf{r}), \Omega\left(\mathbf{r}^{\prime}\right)\right),
$$

where $V_{\alpha \beta}^{(\text {cent })}$ and $V_{\alpha \beta}^{(\text {orient })}$ are respectively the potentials of centrosymmetric interaction and orientational one; $\Omega(\mathbf{r})$ is the set of Euler angles which describes the orientation of the associate located at the point $\mathbf{r}$.

Let us suppose that a non-directed centrosymmetric interaction is significant for only associates being nearest neighbors. Than, introducing the designation $J_{\alpha \beta}=V_{\alpha \beta}^{(c e n t)} / T$, one can write

$$
J_{\alpha \beta}\left(\mathbf{r}-\mathbf{r}^{\prime}\right)=\frac{E_{\alpha \beta}}{T} \sigma\left(\mathbf{r}-\mathbf{r}^{\prime}\right),
$$

where $\sigma\left(\mathbf{r}-\mathbf{r}^{\prime}\right)=1$ for nearest neighbors and $\sigma\left(\mathbf{r}-\mathbf{r}^{\prime}\right)=0$ - otherwise. The elements of matrix $E_{\alpha \beta}$ determine the energy of non-directed interaction between associates $\alpha$ and $\beta ; T$ is the temperature expressed in energetic units. Thus, we assume that components of alloy occupy sites of some spatial quasi-lattice, and this simplification makes the description much easier. However, the lattice approximation has one serious disadvantage, namely it is impossible to describe positional ordering (and hence crystallization) in the frames of this approach. Nevertheless, this disadvantage is not important for purposes of this work.
The orientational interaction can be modelled in the following way. Regardless of system specificity, the function $E_{o r}\left(\mathbf{r}, \mathbf{r}^{\prime}\right)$ must have minima at coincidence of orientations of two nearest associates that is under the condition $\Omega(\mathbf{r})=\hat{G} \Omega\left(\mathbf{r}^{\prime}\right)$, where $\hat{G}$ is an element of the symmetry group of the associate. If these minima are sharp enough than the continuum model can be rewritten as the discrete one. In order to do it, one can write

$$
E_{o r}\left(\mathbf{r}, \mathbf{r}^{\prime}\right)=\left\{\begin{array}{ll}
U, & \left|\Omega(\mathbf{r})-\Omega\left(\mathbf{r}^{\prime}\right)\right|<\Delta \Omega \\
0, & \left|\Omega(\mathbf{r})-\Omega\left(\mathbf{r}^{\prime}\right)\right|>\Delta \Omega
\end{array},\right.
$$

where $\Delta \Omega$ is some characteristic spatial angle. Thus, the $U$ and the $\Delta \Omega$ represent "depth" and "width" of the orientational energy respectively. In other words, if the orientations of two nearest associates coincide accurate within the $\Delta \Omega$ than this state has the energy gain which is equal $U$. Then, one can talk that the associates have formed the bond which is like chemical bonds between monomers in polymer chains. It is obvious that the maximal number of bonds of each associate $f$ is determined by maximal order of its multipole moment. Thus, the orientational interaction causes the existence of directed and saturated bonds between associates. If $f>2$ then the formation of largescale branched networks takes place. We restrict ourselves to the case of single type of associates having the ability to form directed bonds. The generalization for a case of several types of such structural units can be easily done if necessary.

In order to describe systems with orientational interaction, it is necessary to take into account the specific features of the interaction in each particular case. However, when one is interested only in thermodynamic and global structural characteristics of the network-forming systems, the description can be carried out in a universal manner with the inclusion of only the most general features of the intermolecular interaction (for example, saturation). In this case, within the statistical model of the system, one can replace the averaging over all the orientations of each associate by the summation of statistical weights of all allowable (for this particular system) configurations of the spatial network; moreover, it will suffice to describe these configurations by global characteristics, such as the total number of units of all chains, the number of ends, etc.

So each microstate of the system can be represented as a spatial network of bonds which consists of sites (atoms and associates) and edges (bonds) connecting them. The local geometry of the network is determined by the set of basis vectors $\mathbf{e}_{i}(i=1, \ldots, f)$ which are directed from each site to the center of an adjacent edge (Fig. 1).

If the site coordinates are characterized by the radius vector $\mathbf{r}$, the coordinates $\mathbf{p}$ of one of the adjacent edges are: $\mathbf{p}=\mathbf{r}+\mathbf{e}_{i}$.

At the midpoint of each edge, we define the scalar real field $\Psi(\mathbf{p})=\Psi\left(\mathbf{r}+\mathbf{e}_{i}^{(\mathbf{r})}\right)$. Here, the $\mathbf{e}_{i}^{(\mathbf{r})}$ designate the basis vectors corresponding to the site $\mathbf{r}$. This field has no direct physical meaning and is introduced as an auxiliary tool. However, different averaged characteristics determined with the use of this field have a real meaning.

For simplicity in representing subsequent relationships in the lattice formulation of the model, it is expedient to 


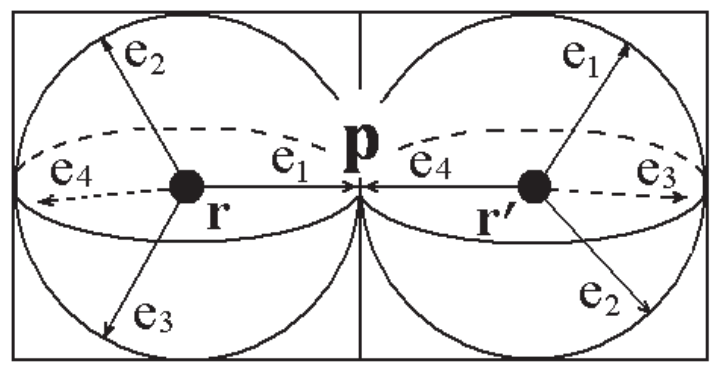

Fig. 1. The schematic picture of two nearest sites of spatial network occupied by associates. The variable $\mathbf{p}$ is specified on the center of edge connected the sites $\mathbf{r}$ and $\mathbf{r}^{\prime}$

change over to indices numbering the sites and edges of the lattice as $r$ and $r+i$, respectively. In this case , the field variables are written in the form $\Psi_{p}=\Psi_{r+i}$.

The effective Hamiltonian of the model is:

$$
H\{n, \Psi\}=H_{l g}\{n\}+H_{0}\{\Psi\}+H_{\text {int }}\{n, \Psi\},
$$

where

$$
\begin{aligned}
& H_{\mathrm{lg}}\{n\}=-\frac{1}{2} \sum_{r r^{\prime}, \alpha \beta} n_{r}^{\alpha} J_{r r^{\prime}}^{\alpha \beta} n_{r^{\prime}}^{\beta}, \\
& H_{0}\{\Psi\}=\frac{K}{2} \sum_{r, i} \Psi_{r+i}^{2}, \\
& H_{\mathrm{int}}\{n, \Psi\}=-\sum_{r} \ln \sum_{\alpha} n_{r}^{\alpha}\left(1+\zeta_{\alpha} P_{r}(\Psi)\right), \\
& P_{r}(\Psi)=a_{1} \sum_{i} \Psi_{r+i}+a_{2} \sum_{i \neq j} \Psi_{r+i} \Psi_{r+j}+\ldots \\
& \quad+a_{f} \sum_{i \neq j \neq \ldots \neq k} \Psi_{r+i} \Psi_{r+j} \ldots \Psi_{r+k} .
\end{aligned}
$$

The term (2) is the Hamiltonian of the generalized model of the lattice gas. The indexes $\alpha, \beta$ enumerate components of the alloy (atoms and their associates), which number is equal $M \geq 2 ; n_{r}$ is the M-component vector formed according to the rule: if the component with the index $\alpha$ is located at the point $r$, then $n_{r}^{\alpha}=\delta_{\alpha \beta}$. This Hamiltonian makes it possible to describe qualitatively correctly the free energy of mixing of the system in the absence of specific interactions. Terms of such a type represent the standard part of the interaction energy in different lattice models [12], [15], [19], [20], [21].

The terms (3) and (4) are introduced to describe directed bonding. Expression (3) is the Hamiltonian of a free field for the variables $\Psi$. Here $K=\exp (-U / T)$ and $U>0$ is the energy of chemical bond between associates.

At last, the term (4) describes interaction between $\Psi$ and $n$, and determines the character of chemical interaction. Here $\zeta$ is the M-component vector formed according to the rule: $\zeta_{\alpha}=1$, if the component $\alpha$ is able to form directed bonds and $\zeta_{\alpha}=0-$ otherwise. The coefficients $a_{m}$ determine statistical weights of $m$-bonded associates; in other words, $\ln a_{m}$ expresses the reduction of the entropy of an associate as result of formation of $m$ bonds.

The partition of the model corresponds to the summation over all configurations of variables $n$ and functional integration over the scalar field $\Psi$ :

$$
Z=\sum_{\{n\}} \int(D \Psi) \exp [-H\{n, \Psi\}] .
$$

where the measure of integration is

$$
D \Psi=(K / 2 \pi)^{\frac{\gamma N}{2}} \prod_{p} D \Psi_{p}
$$

where $\gamma$ is the number of the nearest neighbors for each site and $\gamma N / 2$ is the number of all lattice sites.

As shown in [15], [18], the partition function (6) corresponds to the summation over all possible ways of the arrangement of units over lattice sites and over all possible configurations of chains of chemically bonded associates and ones is taken into account with the correct statistical weights.

Moreover, using (6) as a generating functional, one can obtain different structural averages, for examples the ratio between the number of reacted functional groups and their total number (co called conversion $\Gamma$ ) and the average fraction of bound associates $\Gamma_{b}$.

\section{Mean field approximation}

In order to calculate the thermodynamic characteristics we use the mean-field approximation (MFA). This approximation is the simplest one but, in the majority of cases, it qualitatively correctly describes the behavior of the system.

In the frames of MFA, state of the system is determined by two external parameters: temperature $T$ and concentration $x$ of $\mathrm{B}$ atoms, and $M+1$ internal (variable) parameters, such as $\omega_{\alpha}=\left\langle n_{r}^{\alpha}\right\rangle, \Psi=\left\langle\Psi_{p}\right\rangle$. The $\omega_{\alpha}$ represent mean concentrations of components, and the $\Psi$ is proportional to the mean number of bonds per site.

After simple but rather cumbersome calculations (see [18]), one gets the expression for the specific (per lattice site) free energy of the system:

$$
\begin{aligned}
& F\left(T, x ; \omega_{\alpha}, \Psi\right)=-\frac{\gamma}{2} \sum_{\alpha, \beta} E_{\alpha \beta} \omega_{\alpha} \omega_{\beta}+\frac{\gamma T K}{4} \Psi^{2}+ \\
&+T \sum_{\alpha} \omega_{\alpha} \ln \frac{\omega_{\alpha}}{1+\zeta_{\alpha} R(\Psi)}, \\
& R(\Psi)=a_{1} \gamma \Psi+a_{2} \frac{\gamma(\gamma-1)}{2} \Psi^{2}+\ldots+a_{f} C_{\gamma}^{f} \Psi^{f},
\end{aligned}
$$

where $C_{\gamma}^{f}=\gamma ! / f !(\gamma-f)$ !.

The variables $\omega_{\alpha}$ must satisfy to the material balance equations

$$
\sum_{\alpha} \omega_{\alpha}=1, \quad \frac{\sum_{\alpha} q_{\alpha} \omega_{\alpha}}{\sum_{\alpha}\left(p_{\alpha}+q_{\alpha}\right) \omega_{\alpha}}=x
$$


where $x=N_{b} /\left(N_{a}+N_{b}\right)$ is the concentration of B atoms in the alloy. The equations (9) show, that only $(M-2)$ of $M$ variables $\omega_{\alpha}$ are independent. This implies that these $(M-2)$ variables must be expressed as functions of external parameters $(x, T)$ by means of the chemical equilibrium equations:

$$
p_{\alpha} \tilde{\mu}_{A_{1}}+q_{\alpha} \tilde{\mu}_{B_{1}}-\tilde{\mu}_{A_{p_{\alpha}} B_{q_{\alpha}}}=\Delta G_{A_{p} B_{q}}^{(0)}(T),
$$

where $\Delta G_{A_{p} B_{q}}^{(0)}(T)$ is the Gibbs free energy of formation of the associate $A_{p} B_{q}$ and $\tilde{\mu}_{k}$ are the excess chemical potentials which can be easily found from (7) by standart formulas [22].

The equilibrium equation for the variable $\Psi$ is obtained from the condition $\partial f / \partial \Psi=0$ :

$$
\frac{\gamma e^{-\frac{U}{T}}}{2} \Psi=\omega_{0} \frac{R^{\prime}(\Psi)}{1+R(\Psi)},
$$

where $\omega_{0}$ is the concentration of associates which forms directed bonds.

Thus the expressions (7),(10) and (11) compose the self-contained system of equation permitting to derive all the thermodynamic and structural properties in the MFA.

\section{The case of single associate $A_{2} B$ type}

\subsection{The model assumptions}

Let us consider the important special case of the system which consists of free atoms $A, B$ and the single element of CSRO with the stoichiometry $A_{2} B$. We guess that this type of associates serves as the universal structural unit forming a spatial network. The physical background of this idea can be found in [16], [17].

In order to calculate the Gibbs free energy of formation of the associate $A_{2} B$ we use the simplest approximation $\Delta G^{(0)}(T)=\Delta H^{(0)}-T \Delta S^{(0)}$, where $\Delta H^{(0)}$ and $\Delta S^{(0)}$ do not depend on temperature. The analysis of experimental dates shows that such behavior is typical one [23].

In the following, we will use reduced variables such as $\tilde{T}=T / U, \tilde{E}_{\alpha \beta}=E_{\alpha \beta} / U, \Delta \tilde{H}^{(0)}=\Delta H^{(0)} / U$ and so on. This implies that all the energies and temperature will be measured in the unit of the chemical interaction energy $U$.

The strict determination of the parameters $a_{m}$ requires to calculate the cutting down of orientational degrees of freedom of an associate by formation of $m$ bonds. It is extreme difficult to solve this problem exactly but it is qualitatively clear that $a_{j+1}<a_{j}$. Then, if the network forming structural units are associates with a specified functionality, we can naturally set $a_{m}=b^{m}$. So each bond between two associates (apart from the energy gain $U$ ) corresponds to a loss entropy $2 \ln b$. The parameter $b(0<b \leq 1)$ characterizes the rigidity of the chemical bond: the smaller the parameter $b$, the stronger the restriction of the degrees of freedom of associates upon formation of bonds (the large the decrease in the entropy). According to the physical meaning, parameter $b$ should tend to zero with a decrease in temperature. By choosing parameter $b(T)$ in the form of exponential function, we set

$$
a_{m}(T)=b^{m}(T)=\rho^{m} e^{-m \frac{\theta}{T}} .
$$

It should be noted that, here, $\rho$ is the determining parameter, whereas the coefficient $\theta$ and the form of the function $b(T)$ do not qualitatively affect the results. Indeed, at high temperatures, we have $b(T) \approx \rho$, and, at low temperatures the contribution of the entropy terms becomes negligible as compared to that of the energy terms.

\subsection{Phase diagram of the model}

Let us examine thermodynamic properties of the studied model. The straightforward calculations of the chemical potentials at different temperatures and concentrations show that decreasing of the temperature causes non-ideality of thermodynamic characteristics because of increasing of the association degree. Furthermore, the concentration dependencies of the chemical potentials become nonmonotonic ones that represents the occurrence of layering in the system. So it is interesting to calculate and analyze the typical phase diagram of the model. In order to do so the standart procedure can be applied [22]. First of all, using the (7), (10), and (11) one calculates the dependencies $\mu_{A}(T, x)$, $\mu_{B}(T, x)$ of component chemical potentials on temperature and concentration. Then, with the help of the phase equilibrium conditions

$$
\mu_{A}\left(T, x^{(1)}\right)=\mu_{A}\left(T, x^{(2)}\right), \quad \mu_{B}\left(T, x^{(1)}\right)=\mu_{B}\left(T, x^{(2)}\right),
$$

one can easily get the phase coexistence lines $x^{(1)}(T)$ and $x^{(2)}(T)$ representing the so called binodal. The typical result of such calculations is shown in Fig. 2, and presents two immiscibility gap meeting in the point $\mathrm{D}$ which is called a point of equal concentration [24]. At such a point, in contrast to the critical one, two distinct phases coexist.

This kind of diagram represents a typical fragment of a fusibility curve for metallic alloys in the presence of a chemical compound [25]. In this case, the external binodal in Fig. 2 corresponds to a liquidus line on a real phase diagram, and the point $\mathrm{D}$ is usually called a distectic one [22]. Hereinafter, we will abide by this terminology calling lines and points of the calculated diagram as these ones in correspondence fusibility curve of real metallic alloys.

The concentration corresponding to a distectic point is invariant with respect to values of model parameters and is determined by the stoichiometry of the associate (for $A_{2} B, x_{D}=1 / 3$ ). Variations of model parameters allow to fit fusibility curve of a real diagram exactly. As a rule, the compound homogeneity region of real systems is vanishingly small and presented by a vertical line on the phase diagram. Such a situation is typical for metal-metalloid and Al-RE alloys considering in this work [25]. This is due to crystallization of the chemical compound which eliminates solubility. Since the crystallization is not accounted by the model, the compound homogeneity region can not be fitted exactly. 


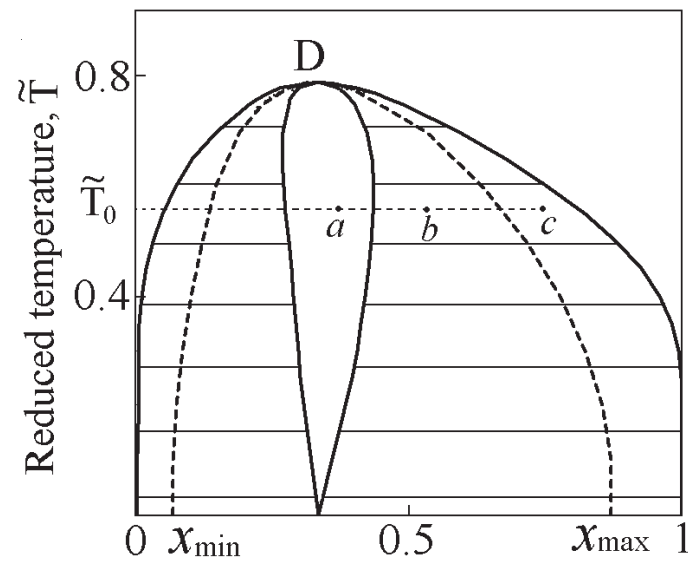

Concentration of B atoms, $x$

Fig. 2. Typical phase diagram and the sol gel transition line of the system which consists of free atoms and associates with the stoichiometry $A_{2} B$. The crossed areas correspond to a two-phase states. The solid lines are the binodals; dashed line represents the percolation one; $\mathrm{D}$ is a distectic point. The points a,b and c represent the characteristic states exhibiting three different scenarios of sol-gel transition.

In reality, the lines outgoing from a distectic point finally terminate in eutectic or peritectic points. In the model, the lines of the diagram continue uninterruptedly down to indefinitely small temperatures and till arbitrary concentrations which is due to crystallization neglecting also. However, these disadvantages are essential only for calculation of equilibrium phase diagrams of real systems that is not the aim of this work. Crystalline ordering is kinetically suppressed by rapid quenching so as the calculated phase diagram can be treated as a metastable one.

\subsection{Structural properties and sol-gel transition}

Let us consider the structural characteristics of the model. In order to analyze the global structure of spatial network it is useful to introduce the so-called conversion $\Gamma$ determined as the ratio between the number of reacted functional groups and their total number. In the frames of MFA this quantity can be expressed by the relationsip [18]:

$$
\Gamma(T, x)=\frac{\gamma e^{-\frac{U}{T}}}{2 f \omega_{0}(T, x)} \Psi^{2}(T, x),
$$

where $\omega_{0}(T, x)$ and $\Psi(T, x)$ are the equilibrium values of these quantities.

The detailed analysis $\Gamma(T, x)$ at different concentrations and temperatures shows that in the homogeneous region, that is at $T>T_{L}$ (where $T_{L}$ corresponds to a liquidus temperature), this quantity is very small and slowly increase with decreasing temperature. This implies that the alloy contains only short faintly branched networks. In other words, the state of the system is near to an ideal associated solution. However, at $T<T_{L}$ the quantities $\chi(T)$ and $v(T)$ begin to rise sharply that is the evidence of intensive growth of a spatial network. At some temperatures and concentrations, this process can result in geometry percolation of the network and thus a sol-gel transition may take place.

In order to determine the criterion for the onset of the percolation it is necessary to calculate the correlation function $G\left(r-r^{\prime}\right)$ for the monomers belonging to the connected cluster, i.e. for two sites joined by a connected path of chemical bonds. The criterion for the formation of the infinite percolation cluster will be a divergence of the correlation length $r_{c}$ of the function $G$. If the $r_{c}$ will be calculated as a function of the temperature $T$ an concentration $x$, this condition will determine the equation of the percolation line in the $T-x$ plane. According to calculations made in [18], the equation of the critical percolation line has the form:

$$
\Gamma(T, x) \omega_{0}(T, x)=\alpha .
$$

Here $\alpha=\gamma z(\lambda) / 2 f$ is the constant determined by the lattice geometry and the functionality of monomers; $\lambda=\left(R_{2} / R_{1}\right)^{3}$ is the geometric factor determining the packing density of particles; $R_{1}$ and $R_{2}$ are the radii of the first and second coordination shells respectively; $z(\lambda)$ is the real solution of cubic equation

$$
1-\frac{5}{4} \lambda z+z^{2}-\frac{1}{8} \lambda z^{3}=0 .
$$

It can be seen from (14) that the behavior of sol-gel transition line is completely determined by the dependencies of the $\Gamma$ and $\omega_{0}$ on the temperature and concentration. The analysis of that dependencies and the equation (14) demonstrates the next qualitative picture (see Fig. 4.2).

The percolation line always lies in the two-phase area and its maximum coincides with the distectic point. So the competition between gelation and phase separation can take place. In addition, at $T=0$ percolation line terminates in two characteristic concentrations $x_{\min }$ and $x_{\text {max }}$. This implies that, at $x<x_{\min }$ or $x>x_{\max }$, the percolation transition is not observed at any temperatures. However, the later remark attributes to only stable state since in two-phase area gelation can occur at $x<x_{\min }$ or $x>x_{\max }$ due to the phase separation.

Let the system is quenched to some temperature $\tilde{T}_{0}$ at three different concentrations (see points a, b, c in Fig. 4.2 ). Then three different scenarios will realize:

a. If the system is quenched to one-phase area under cupola (point (a) Fig. 4.2) then the sol-gel transition will occur continuously without any thermodynamic manifestations.

b. If the system is quenched to the area corresponding two-phase states and lying under percolation line (point (b) Fig. 4.2) then gel state is unstable and phase separation follows gelation. It should be noted that if the temperature $\tilde{T}_{0}$ is small enough and therefore viscosity of the gel has large value then phase separation will be impeded. In this case system may even undergo dynamical arrest and freeze in a nonequilibrium state.

c. Finally, if the system turns out in the two phase area but outside percolated region (point (c) Fig. 4.2) then the sol-gel transition will be accompanied by the phase 
separation; i.e. it will proceed as a first-order phase transition. In this case dynamical arrest is also possible.

The aforementioned scenarios should be taken into account on investigation of phase separation and amorphization in glass-forming alloys but detailed consideration of these questions is the subject of separate work.

\section{Conclusions}

In this paper, we have applied the statistical model for describing network-forming systems, developed in our previous works, to study of metallic alloys with chemical bonding. The model is based on the representation of the sum of statistical weights over all possible configurations for a thermoreversible network in the form of a functional integral over a scalar field. The main advantages of the proposed approach are mathematical rigor, universality and flexibility.

The main object of our investigation was the solution of free atoms $A, B$ and the elements of chemical shortrange order with the stoichiometry $A_{2} B$ that could form thermoreversible chemical bonds. We guess that these associates serve as structural network-forming units in metalmetalloid and Al-REM glass-forming alloys. We analyzed the thermodynamic and structural properties of this system. The general results of this analysis are presented in Fig. 4.2.

The typical phase diagram of the system under consideration represents two immiscibility gap meeting in the distectic point. This kind of diagram is a typical fragment of a fusibility curve for real metallic alloys in the presence of a chemical compound.

The analysis of structural properties of the system shows that at some temperatures and concentrations, geometry percolation of the network of chemical bonds and thus a sol-gel transition may take place. The critical percolation line was plotted in common with phase diagram. Three different scenarios of sol-gel transition was considered in light of competition between gelation and phase separation phenomena.

The obtained results can play an impotent role in physics of metal-metalloid and Al-REM alloys for investigation of its glass-forming ability, layering processes and quenching regimes.

This work was supported by the Russian Foundation for Basic Research (project no. 10-02-00882-à).

\section{References}

1. L.-M. Martinez, C.A. Angell, Physica A 314, 548 (2002)

2. P.G. Debenedetti, J. Phys.: Condens. Matter 15, R1669 (2003).

3. M. Gordon, S.B. Ross-Murphy, Pure Appl. Chem. 43, 1 (1975).
4. S.K. Kumar, A.Z. Panagiotopoulos, Phys. Rev. Lett. 82, 5060 (1999).

5. de Greef-Meijer, Sangeetha T.F.A. de Greef, E.W. Meiler Nature 453, 171 (2008)

6. N.M. Sangeetha, U. Maitra Chem. Soc. Rev. 34, 821 (2005)

7. E. Zaccarelli, J. Phys.: Condens. Matter 19323101 (2007).

8. A.G. Zilman, S.A. Safran, Phys. Rev. E 66, 051107 (2002)

9. F.Tanaka, A. Matsuyama, Phys. Rev. Lett. 62, 2759 (1989)

10. F. Tanaka, W.H. Stockmayer, Macromolecules 27, 3943 (1994)

11. A.N. Semenov, M. Rubinstein, Macromolecules, 31, (1998) 1373

12. A. Coniglio, H.E. Stanley, W. Klein, Phys. Rev. Lett. 42, 518 (1979); Phys. Rev. B 25, 6805 (1982)

13. I. Erukhimovich, M.V. Thamm, A.V. Ermoshkin, Macromolecules, 34, 5653 (2001)

14. T.C. Lubensky, J. Isaacson, Phys. Rev. Lett. 41, 829 (1978); Phys. Rev. A 20, 2130 (1979)

15. R.E. Ryltcev, L.D. Son, Physica A 368, 101 (2006)

16. L.D. Son, R.E. Ryltcev, V.E. Sidorov, D. Sordelet, Mater. Sci. Eng. A 449-451, 582 (2007)

17. V.E. Sidorov et al., Mater. Sci. Eng. A 449-451, (2007) 586

18. R.E. Ryltsev, L.D. Son, JETP 110, 504 (2010)

19. S. Sastry, P.G. Debenedetti, F. Sciortino, H.E. Stanley, Phys. Rev. E 53, 6144 (1996)

20. G. Franzese, M.I. Marque, H.E. Stanley, Phys. Rev. E 67, 011103 (2003)

21. A.Yu. Zakharov, M.A. Zakharov, V.V. Lebedev, Int. J. of Quant. Chem. 104, 126 (2005)

22. I. Prigogine, R. Defay, Chemical thermodynamic (Longmans Green and Co, London-NY-Toronto, 1954)

23. I. Barin, O. Knacke, Termochemical properties of inorganic substances (Springer-Verlag, Berlin, 1973)

24. L.D. Landau, E.M. Lifshitz, Course of theoretical physics. V5. Statistical physics (Pergamon Press, 1980)

25. T.B. Massalski, Binary alloys phase diagrams (American society for metals, Ohio, 1987) 